Int. Journal of Math. Analysis, Vol. 7, 2013, no. 4, 155 - 174

\title{
On a Class of Nonlocal Degenerate Reaction-Diffusion Equations with Localized Nonlinear Diffusion Term
}

\author{
Sikiru Adigun Sanni \\ Department of Mathematics \& Statistics \\ University of Uyo \\ Uyo 520003, Nigeria \\ sikirusanni@yahoo.com
}

\begin{abstract}
We study a class of second order nonlocal degenerate semilinear reaction-diffusion equations with localized nonlinear diffusion term. Under a set of conditions on the localized nonlinear diffusivity and nonlinear nonlocal source term, we prove global existence and uniqueness result in the whole of some weighted Sobolev's spaces. Furthermore, we prove nonexistence of smooth solution or blow-up of solution under some other set of conditions. Finally, we give illustrative examples for which our results apply.
\end{abstract}

Mathematics Subject Classification: Primary 35K05, 35K10, 35K20, $35 \mathrm{~K} 58,35 \mathrm{~K} 65$

Keywords: initial boundary value problems; Galerkin's approximations; energy estimates; Banach's fixed point theorem; existence and uniqueness of weak solutions

\section{Introduction}

We consider the following class of degenerate semilinear parabolic second order initial boundary value problems:

$$
\begin{aligned}
u_{t}-\left(\phi_{u}(t, x) u_{x}\right)_{x} & =f(u), \text { in }(0, T] \times(0, a) \\
u(t, 0) & =0, u(t, a)=0, \text { in }(0, T] \\
u(0, x) & =g(x), x \in(0, a),
\end{aligned}
$$


where $g \in L^{2}(0, a)$; the nonsmooth nonlinearities $\phi_{u}(t, x):[0, a] \rightarrow \mathbb{R}$ and $f(u): \mathbb{R} \rightarrow \mathbb{R}$ satisfy some combinations of the following conditions, namely:

$$
\begin{aligned}
\gamma u^{p} & \leq f(u), \text { for some constant } p>1 \\
\phi_{u}(t, x) & \leq \beta \psi(x) \\
\lambda_{1} \psi(x) \leq \phi_{u}(t, x) & \leq \lambda_{2} \psi(x), \quad\left(\lambda_{1} \geq 1,3 \lambda_{1}-\lambda_{2}>0\right), \\
\left|\phi_{u}(t, x)-\phi_{v}(t, x)\right| & \leq L_{1} \psi(x) \int_{0}^{x}|u-v| d s \\
|f(u)-f(v)| & \leq L|u-v|
\end{aligned}
$$

for some continuous functions $\psi \geq 0$ and some positive constants $\gamma, \beta, \lambda_{2}, L_{1}$ and $L$.

Second order parabolic equations describe the time-evolution of the density of some physical quantity $u$, say chemical concentration, temperature or electric potential, etc.

If $\phi_{u}>0,(1)$ corresponds to nondegenerate reaction-diffusion equations with nonlocal source. The nondegenerate reaction-diffusion equations with nonlocal source has been considered by several authors, see for example $[2$, $5,14,15,16,17,19,21,22]$. Authors who have investigated nondegenerate reaction-diffusion equations with local source terms include Cazenave and Lions [6], Friedman and Mcleod [12], Giga and Kohn [13], and Ni et al. [15]. Among several authors who have investigated degenerate reaction-diffusion equations are Budd et. al. [3], Budd et. al. [4], Chen et. al. [8], Chun and Li [7], Floater [11], and Souplet [19, 20]. The latter mentioned authors are concerned with the blow-up properties of the solutions to the various problems considered.

In a recent paper, Chen and Lihua [9] considered (1)-(3) with the particular degenerate diffusion term $\left(x^{\alpha} u_{x}\right)_{x}(0<\alpha<1)$ and the local source term $b f\left(u\left(x_{o}(t), t\right)\right)$, where $b>0$. The authors show that, under certain conditions, global solutions exist for small $b$ or small initial data; while the solutions blow up for large $b$ or large initial data. Furthermore, the authors show that all global solutions are uniformly bounded. Motivated by the work of Chen and Lihua [6], Sanni [18] considered (1)-(3) with the general diffusion term $\left(\phi(x) u_{x}\right)_{x}$ and the nonlocal source term $f(u)$. Under some conditions on the diffusion and the source terms the author proved the existence and uniqueness of global weak solutions to the class of semilinear degenerate problems in some weighted Sobolev's spaces.

The current work is an improvement on the paper [18]. The introduction of the localized nonlinear degenerate diffusion term introduces more difficulty in the analysis than in [18]. Under a set of conditions on the localized nonlinear diffusivity and nonlinear nonlocal source term, we prove global existence and uniqueness result in the whole of some weighted Sobolev's spaces. Further- 
more, we prove nonexistence of smooth solution or blow-up of solution under another set of conditions.

The remaining part of this paper is organized as follows. In Section 2, we define the spaces used in this paper, give the definition of our weak solution and state some known theorems. In Section 3, we constructed Galerkin's approximations for an auxiliary linear problem, obtained energy estimates and proved the existence of a unique weak solution to the linear problem. The existence of unique weak solutions to the problems (1)-(3) is proved in subsection 4.1. The nonexistence of smooth solution or blow up of solution is proved in subsection 4.2. In Section 5, we give illustrative examples for which our results are applicable.

\section{Preliminaries}

We adopt the idea of not considering $u$ as a function of $x$ and $t$, but rather as a mapping

$$
\mathbf{u}:[0, T] \rightarrow L^{2}(0, a) \cap H_{0}^{1}((0, a), \psi(x))
$$

defined by

$$
[\mathbf{u}(t)](x):=u(t, x) \quad(x \in(0, a), t \in[0, T]) .
$$

Definition 2.1. Let

$L^{\infty}\left[0, T ; L^{2}(0, a)\right]:=$ The space of all measurable functions $u:[0, T] \rightarrow$ $L^{2}(0, a)$ with the norm

$$
\|\mathbf{u}\|_{L^{\infty}\left[0, T ; L^{2}(0, a)\right]}:=\operatorname{ess} \sup \|\mathbf{u}\|_{L^{2}(0, a)}<\infty .
$$

Let

$L^{2}((0, a), \psi(x)):=\left\{\mathbf{u}:(0, a) \rightarrow \mathbb{R}\right.$ such that $\left.\|\mathbf{u}\|_{L^{2}((0, a), \psi(x))}<\infty\right\}$ with the norm

$$
\|\mathbf{u}\|_{L^{2}((0, a), \psi(x))}:=\sqrt{\int_{0}^{a} \psi(x) \mathbf{u}^{2} d x} .
$$

Let

$H^{1}((0, a), \psi(x)):=\left\{\mathbf{u}:(0, a) \rightarrow \mathbb{R}\right.$ such that $\left.\|\mathbf{u}\|_{H^{1}((0, a), \psi(x))}<\infty\right\}$ with the norm

$$
\|\mathbf{u}\|_{H^{1}((0, a), \psi(x))}:=\sqrt{\int_{0}^{a} \psi(x) \mathbf{u}^{2} d x+\int_{0}^{a} \psi(x) \mathbf{u}_{x}^{2} d x} .
$$

Let

$H_{0}^{1}((0, a), \psi(x)):=$ the closure of the $C_{c}^{\infty}((0, a), \psi(x))$ in $H^{1}((0, a), \psi(x))$, with the norm

$$
\|\mathbf{u}\|_{H_{0}^{1}((0, a), \psi(x))}:=\sqrt{\int_{0}^{a} \psi(x) \mathbf{u}_{x}^{2} d x} .
$$


Let

$L^{2}\left[0, T ; H_{0}^{1}((0, a), \psi(x))\right]:=$ The space of all measurable functions

$$
u:[0, T] \rightarrow H^{1}((0, a), \psi(x))
$$

with the norm

$$
\|\mathbf{u}\|_{L^{2}\left[0, T ; H_{0}^{1}((0, a), \psi(x))\right]}:=\sqrt{\int_{0}^{T}\|\mathbf{u}\|_{H_{0}^{1}((0, a), \psi(x))}^{2} d t}<\infty .
$$

Let

$D_{\psi}:=L^{2}(0, a) \cap H_{0}^{1}((0, a), \psi(x))$ with the norm

$$
\|\mathbf{u}\|_{D_{\psi}}:=\sqrt{\|\mathbf{u}\|_{L^{2}(0, a)}^{2}+\|\mathbf{u}\|_{H_{0}^{1}((0, a), \psi(x))}^{2}} .
$$

Let

$Y_{\psi}:=L^{\infty}\left[0, T ; L^{2}(0, a)\right] \cap L^{2}\left[0, T ; H_{0}^{1}((0, a), \psi(x))\right]$ with the norm

$$
\|\mathbf{u}\|_{Y_{\psi}}:=\sqrt{\|\mathbf{u}\|_{L^{\infty}\left[0, T ; L^{2}(0, a)\right]}^{2}+\|\mathbf{u}\|_{L^{2}\left[0, T ; H_{0}^{1}((0, a), \psi(x))\right]}^{2}} .
$$

Let

$L^{2}\left[0, T ; D_{\psi}^{-1}\right]:=$ The space of all measurable functions $\mathbf{u}:[0, T] \rightarrow D_{\psi}^{-1}$ with the norm

$$
\|\mathbf{u}\|_{L^{2}\left[0, T ; D_{\psi}^{-1}\right]}:=\sqrt{\int_{0}^{T}\|\mathbf{u}\|_{D_{\psi}^{-1}}^{2} d t}<\infty,
$$

where $D_{\psi}^{-1}$ is the dual space of $D_{\psi}$.

Definition 2.2. A function

$$
\mathbf{u} \in Y_{\psi}, \text { with } \mathbf{u}^{\prime} \in L^{2}\left[0, T ; D_{\psi}^{-1}\right]
$$

is a weak solution of the degenerate parabolic initial boundary value problem (1)-(3) provided

$$
\int_{0}^{a} \mathbf{u}^{\prime} v d x+\int_{0}^{a} \phi_{\mathbf{u}}(t, x) \mathbf{u}_{x} v_{x} d x=\int_{0}^{a} f(\mathbf{u}) v d x
$$

for each $v \in D_{\psi}$ and a.e. time $0 \leq t \leq T$,

$$
\mathbf{u}(0)=g
$$

where $g \in L^{2}(0, a)$.

We will need the following theorem proved in [18]. 
Theorem 2.3. Let $\mathbf{u} \in Y_{\psi}$ with $\mathbf{u}^{\prime} \in L^{2}\left[0, T ; D_{\psi}^{-1}\right]$.

(i) Then

$$
\mathbf{u} \in C\left[0, T ; L^{2}(0, a)\right]
$$

(after possibly being redefined on a set of measure zero).

(ii) The mapping

$$
t \mapsto\|\mathbf{u}(t)\|_{L^{2}(0, a)}^{2}
$$

is absolutely continuous, with

$$
\frac{d}{d t}\|\mathbf{u}(t)\|_{L^{2}(0, a)}^{2}=2\left\langle\mathbf{u}^{\prime}(t), \mathbf{u}(t)\right\rangle
$$

for a.e. $0 \leq t \leq T$

(iii) Furthermore, we have the estimate

$$
\max _{0 \leq t \leq T}\|\mathbf{u}(t)\|_{L^{2}(0, a)} \leq C\left(\|\mathbf{u}(t)\|_{Y_{\psi}}+\left\|\mathbf{u}^{\prime}(t)\right\|_{L^{2}\left[0, T ; D_{\psi}^{-1}\right]}\right)
$$

where $C=C(T)$.

\section{$3 \quad$ Auxiliary linear problem}

Consider the following degenerate linear parabolic initial boundary value problems:

$$
\begin{aligned}
u_{t}-\left(\phi_{s}(t, x) u_{x}\right)_{x} & =f(s(t, x)), \text { in }(0, T] \times(0, a) \\
u(t, 0) & =0, u(t, a)=0, \text { in }(0, T] \\
u(0, x) & =g(x), x \in(0, a),
\end{aligned}
$$

where $s \in L^{2}\left[0, T ; L^{2}(0, a)\right]$ is a known function.

Remark 3.1. The condition $s \in L^{2}\left[0, T ; L^{2}(0, a)\right]$ and the Lipschitz's condition on $f(s)$ imply that

$$
\|f(s)\|_{L^{2}\left[0, T ; L^{2}(0, a)\right]} \leq C(L,|f(0)|, T)\left(1+\|s\|_{L^{2}\left[0, T ; L^{2}(0, a)\right]}\right)<\infty,
$$

so that $f(s) \in L^{2}\left[0, T ; L^{2}(0, a)\right][18]$.

The definition of our weak solution for $(14)-(16)$ is the same as in the Definition 2.2 with $\phi_{\mathbf{u}}(t, x)$ and $f(\mathbf{u})$ replaced by $\phi_{s}(t, x)$ and $f(s)$ respectively.

We shall build a weak solution of our degenerate parabolic problem (14)-(16) by constructing some finite-dimensional approximations (Galerkin approximations), before passing to limits. 
Assume that the functions $w_{k}=w_{k}(x)(k=1, \ldots)$ are smooth,

$$
\begin{aligned}
& \left\{w_{k}\right\}_{k=1}^{\infty} \text { is an orthogonal basis of } H_{0}^{1}\left((0, a), \phi_{s}(t, x)\right) \text { and } \\
& \left\{w_{k}\right\}_{k=1}^{\infty} \text { is an orthonormal basis of } L^{2}(0, a) .
\end{aligned}
$$

(We can for example take $\left\{w_{k}\right\}_{k=1}^{\infty}$ to be the complete set of appropriately normalized eigenfunctions for $-\frac{\partial}{\partial x}\left(\phi_{s}(t, x) \frac{\partial}{\partial x}\right)$ in $\left.H_{0}^{1}\left((0, a), \phi_{s}(t, x)\right)\right)$

We fix a positive integer $\mathrm{m}$; and look for function

$$
\mathbf{u}^{m}:[0, T] \rightarrow H_{0}^{1}\left((0, a), \phi_{s}(t, x)\right)
$$

of the form

$$
\mathbf{u}^{m}:=\sum_{k=1}^{m} d_{m}^{k}(t) w_{k},
$$

where we intend to select the coefficients $d_{m}^{k}(t)(0 \leq t \leq T), k=1, \ldots, m$ so that

$$
\begin{array}{r}
d_{m}^{k}(0)=\int_{0}^{a} g w_{k} d x,(k=1, \ldots, m), \text { and } \\
\int_{0}^{a} \mathbf{u}^{m \prime} w_{k} d x+\int_{0}^{a} \phi_{s}(t, x) \mathbf{u}_{x}^{m}\left(w_{k}\right)_{x} d x=\int_{0}^{a} f(s) w_{k} d x
\end{array}
$$

The proof of the following theorem is precisely the same as in [18] with $\phi(x)$ replaced by $\phi_{s}(t, x)$.

Theorem 3.2. (Construction of approximate solutions). There exists a unique function $\mathbf{u}^{m}$ of the form (20) satisfying (21)-(22) for each integer $m=1,2, \ldots$.

Theorem 3.3. (Energy estimates). Let the conditions (6)-(8) be satisfied. Then there exists a constant $C>0$ such that

$$
\begin{aligned}
& \sup _{0 \leq t \leq T}\left\|\mathbf{u}^{m}(t)\right\|_{L^{2}(0, a)}^{2}+\left\|\mathbf{u}^{m}\right\|_{L^{2}\left[0, T ; H_{0}^{1}((0, a), \psi(x))\right]}^{2}+\left\|\mathbf{u}^{m \prime}\right\|_{L^{2}\left[0, T ; D_{\psi}^{-1}\right]}^{2} \\
& \leq C\left(\|g\|_{L^{2}(0, a)}+\|s\|_{L^{2}\left[0, T ; L^{2}(0, a)\right]}+1\right)^{2}
\end{aligned}
$$

for $m=1,2, \ldots$; where $C=C\left(T, L, \lambda_{1}, \lambda_{2},|f(0)|\right)$.

Proof. We split the proof in two steps.

Step 1. Multiplying equation $(22)$ by $d_{m}^{k}(t)$, summing over $k=1, \ldots, m$, recalling (20) and using (6), we deduce

$$
\begin{aligned}
\frac{d}{d t}\left(\int_{0}^{a}\left(\mathbf{u}^{m}\right)^{2} d x\right) & +2 \lambda_{1} \int_{0}^{a} \psi(x)\left(\mathbf{u}_{x}^{m}\right)^{2} d x \leq 2 \int_{0}^{a} f(s) \mathbf{u}^{m} d x \\
& \leq \int_{0}^{a}\left(\mathbf{u}^{m}\right)^{2} d x+\int_{0}^{a} f(s)^{2} d x
\end{aligned}
$$


Notice that (24) implies that

$$
\frac{d}{d t}\left(\int_{0}^{a}\left(\mathbf{u}^{m}\right)^{2} d x\right) \leq \int_{0}^{a}\left(\mathbf{u}^{m}\right)^{2} d x+\int_{0}^{a} f(s)^{2} d x .
$$

Applying Gronwall's inequality to (25), we deduce

$$
\sup _{[0, T]}\left\|\mathbf{u}^{m}\right\|_{L^{2}(0, a)}^{2} \leq C(T)\left(\int_{0}^{a} g^{2} d x+\int_{0}^{T} \int_{0}^{a} f^{2}(s) d x d t\right) .
$$

Using (17) in (26), we deduce

$$
\sup _{[0, T]}\left\|\mathbf{u}^{m}\right\|_{L^{2}(0, a)}^{2} \leq C(T, L,|f(0)|)\left(\|g\|_{L^{2}(0, a)}+\|s\|_{L^{2}\left[0, T ; L^{2}(0, a)\right]}+1\right)^{2}
$$

Integrating (24) over $[0, T]$ and employing (27), we deduce

$$
\left\|\mathbf{u}^{m}\right\|_{L^{2}\left[0, T ; H_{0}^{1}((0, a), \psi(x))\right]}^{2} \leq C\left(T, L, \lambda_{1},|f(0)|\right)\left(\|g\|_{L^{2}(0, a)}+\|s\|_{L^{2}\left[0, T ; L^{2}(0, a)\right]}+1\right)^{2}
$$

Step 2. Now, fix any $v \in D_{\phi_{s}}$, such that $\|v\|_{D_{\phi_{s}}} \leq 1$, and set $v=v^{1}+v^{2}$, where $v^{1} \in \operatorname{span}\left\{w_{k}\right\}_{k=1}^{m}$ and $\int_{0}^{a} v^{2} w_{k} d x=0(k=1, \ldots, m)$. Thus $\left\|v^{1}\right\|_{D_{\phi_{s}}} \leq$ $\|v\|_{D_{\phi_{s}}} \leq 1$, since $\left\{w_{k}\right\}_{k=0}^{\infty}$ are orthogonal in $H_{0}^{1}\left((0, a), \phi_{s}(t, x)\right)$.

Hence, using (22), we deduce for a.e. $0 \leq t \leq T$ that

$$
\int_{0}^{a} \mathbf{u}^{m \prime} v^{1} d x+\int_{0}^{a} \phi_{s}(t, x) \mathbf{u}_{x}^{m} v_{x}^{1} d x=\int_{0}^{a} f(s) v^{1} d x .
$$

Thus, (20) implies

$$
\begin{aligned}
\left\langle\mathbf{u}^{m \prime}, v^{1}\right\rangle & =\int_{0}^{a} \mathbf{u}^{m \prime} v d x=\int_{0}^{a} \mathbf{u}^{m \prime} v^{1} d x=\int_{0}^{a} f(s) v^{1} d x-\int_{0}^{a} \phi_{s}(t, x) \mathbf{u}_{x}^{m} v_{x}^{1} d x \\
& \leq\|f(s)\|_{L^{2}(0, a)}\left\|v^{1}\right\|_{L^{2}(0, a)}+\left\|\mathbf{u}^{m}\right\|_{H_{0}^{1}\left((0, a), \phi_{s}(t, x)\right)}\left\|v^{1}\right\|_{H_{0}^{1}\left((0, a), \phi_{s}(t, x)\right)} \\
& \leq\|f(s)\|_{L^{2}(0, a)}\left\|v^{1}\right\|_{L^{2}(0, a)}+\lambda_{2}\left\|\mathbf{u}^{m}\right\|_{H_{0}^{1}((0, a), \psi(x))}\left\|v^{1}\right\|_{H_{0}^{1}\left((0, a), \phi_{s}(t, x)\right)}
\end{aligned}
$$

using (6). Therefore,

$$
\left|\left\langle\mathbf{u}^{m \prime}, v^{1}\right\rangle\right| \leq C\left(\lambda_{2}\right)\left(\|f(s)\|_{L^{2}(0, a)}+\left\|\mathbf{u}^{m}\right\|_{D_{\psi}}\right),
$$

since $\left\|v^{1}\right\|_{D_{\phi_{s}}} \leq 1$. We thus have

$$
\left\|\mathbf{u}^{m \prime}\right\|_{D_{\psi}^{-1}} \leq\left\|\mathbf{u}^{m \prime}\right\|_{D_{\phi_{s}}^{-1}} \leq C\left(\lambda_{2}\right)\left(\|f(s)\|_{L^{2}(0, a)}+\left\|\mathbf{u}^{m}\right\|_{D_{\psi}}\right),
$$

using (6), so that

$$
\begin{aligned}
\left\|\mathbf{u}^{m \prime}\right\|_{L^{2}\left[0, T ; D_{\psi}^{-1}\right]} & \leq C\left(\lambda_{2}\right)\left(\|\mathbf{u}\|_{Y_{\psi}}+\|f(s)\|_{L^{2}\left[0, T ; L^{2}(0, a)\right]}\right) \\
& \leq C\left(\|g\|_{L^{2}(0, a)}+\|s\|_{L^{2}\left[0, T ; L^{2}(0, a)\right]}+1\right),
\end{aligned}
$$

where $C=C\left(T, L, \lambda_{1}, \lambda_{2},|f(0)|\right)$ and where we have employed (17), (27) and (28). Using (27)-(29), we deduce (23) as desired. 
Theorem 3.4. There exists a unique weak solution of the linear problem (14)(16).

Proof. The proof is split in four steps.

Step 1.The sequence $\left\{\mathbf{u}^{m}\right\}_{m=1}^{\infty}$ is bounded in $L^{2}\left[0, T ; D_{\psi}\right]$, and $\left\{\mathbf{u}^{m \prime}\right\}_{m=1}^{\infty}$ is bounded in $L^{2}\left[0, T ; D_{\psi}^{-1}\right]$, by the energy estimates $(23)$. Thus, there exists a subsequence $\left\{\mathbf{u}^{m_{l}}\right\}_{l=1}^{\infty} \subset\left\{\mathbf{u}^{m}\right\}_{m=1}^{\infty}$ and a function

$$
\mathbf{u} \in L^{2}\left[0, T ; H_{0}^{1}((0, a), \psi(x))\right]
$$

with $\mathbf{u}^{\prime} \in L^{2}\left[0, T ; D_{\psi}^{-1}\right]$, such that

$$
\begin{aligned}
& \mathbf{u}^{m_{l}} \rightarrow \mathbf{u} \text { in } Y_{\psi} \\
& \mathbf{u}^{m_{l^{\prime}}} \rightarrow \mathbf{u}^{\prime} \text { in } L^{2}\left[0, T ; D_{\psi}^{-1}\right]
\end{aligned}
$$

Step 2. Fix an integer $\mathrm{M}$ and choose a function $v \in C^{1}\left[0, T ; D_{\psi}\right]$ of the form

$$
\mathbf{v}=\sum_{k=1}^{M} d^{k}(t) w_{k}
$$

where $\left\{d^{k}\right\}_{k=1}^{M}$ are given smooth functions. Choosing $m \geq M$, summing over $k=1, \ldots, M$, and then integrating with respect to $t$, we get

$$
\int_{0}^{T} \int_{0}^{a} \mathbf{u}^{m \prime} \mathbf{v} d x d t+\int_{0}^{T} \int_{0}^{a} \phi_{s}(t, x) \mathbf{u}_{x}^{m} \mathbf{v}_{x} d x d t=\int_{0}^{T} \int_{0}^{a} f(s) \mathbf{v} d x d t
$$

Using (6), it is not difficult to show that

$$
\left|\int_{0}^{T} \int_{0}^{a} \phi_{s}(t, x)\left(\mathbf{u}_{x}^{m}-\mathbf{u}_{x}\right) \mathbf{v}_{x} d x d t\right| \leq \lambda_{2}\left\|\mathbf{u}_{x}^{m}-\mathbf{u}_{x}\right\|_{Y_{\psi}}\|v\|_{C^{1}\left[0, T ; D_{\psi}\right]}
$$

Now, setting $m=m_{l}$ in (33), employing (34), recalling (30)-(31) and passing to weak limits, we get

$$
\int_{0}^{T} \int_{0}^{a} \mathbf{u}^{\prime} \mathbf{v} d x d t+\int_{0}^{T} \int_{0}^{a} \phi_{s}(t, x) \mathbf{u}_{x} \mathbf{v}_{x} d x d t=\int_{0}^{T} \int_{0}^{a} f(s) \mathbf{v} d x d t
$$

As the functions of the form (32) are dense in $L^{2}\left[0, T ; D_{\psi}\right]$, we see that (35) holds for all functions in this space. We have in particular

$$
\int_{0}^{a} \mathbf{u}^{\prime} v d x+\int_{0}^{a} \phi_{s}(t, x) \mathbf{u}_{x} v_{x} d x=\int_{0}^{a} f(s) v d x
$$

for each $v \in D_{\psi}$ and a.e. $0 \leq t \leq T$. By (i) of Theorem 2.3, we see furthermore that $\mathbf{u} \in C\left[0, T ; L^{2}(0, a)\right]$. 
Step 3. We now prove that $\mathbf{u}(0)=g$. Notice first that (35) is equivalent to

$$
-\int_{0}^{T} \int_{0}^{a} \mathbf{v}^{\prime} \mathbf{u} d x d t+\int_{0}^{T} \int_{0}^{a} \phi_{s}(t, x) \mathbf{u}_{x} \mathbf{v}_{x} d x d t=\int_{0}^{T} \int_{0}^{a} f(s) \mathbf{v} d x d t+\int_{0}^{a} \mathbf{u}(0) \mathbf{v}(0) d x
$$

for each $\mathbf{v} \in C^{1}\left[0, T ; D_{\psi}\right]$ with $V(T)=0$. Similarly, (33) is equivalent to

$$
\begin{aligned}
-\int_{0}^{T} \int_{0}^{a} \mathbf{v}^{\prime} \mathbf{u}^{m} d x d t & +\int_{0}^{T} \int_{0}^{a} \phi_{s}(t, x) \mathbf{u}_{x}^{m} \mathbf{v}_{x} d x d t \\
& =\int_{0}^{T} \int_{0}^{a} f(s) \mathbf{v} d x d t+\int_{0}^{a} \mathbf{u}^{m}(0) \mathbf{v}(0) d x
\end{aligned}
$$

Setting $m=m_{l}$ in (37) and employing once again (30)-(31), we deduce

$-\int_{0}^{T} \int_{0}^{a} \mathbf{v}^{\prime} \mathbf{u} d x d t+\int_{0}^{T} \int_{0}^{a} \phi_{s}(t, x) \mathbf{u}_{x} \mathbf{v}_{x} d x d t=\int_{0}^{T} \int_{0}^{a} f(s) \mathbf{v} d x d t+\int_{0}^{a} g \mathbf{v}(0) d x$

since $\mathbf{u}_{m_{l}}(0) \rightarrow g \in L^{2}(0, a)$. Comparing (36) and (38), we conclude that $\mathbf{u}(0)=g$, as $\mathbf{v}(0)$ is arbitrary.

Step 4. Finally, we prove uniqueness. Let $\mathbf{u}$ and $\overline{\mathbf{u}}$ be two solutions of (14)(16). Then $\mathbf{u}-\overline{\mathbf{u}}$ satisfies

$$
\begin{array}{r}
\int_{0}^{a}(\mathbf{u}-\overline{\mathbf{u}})^{\prime} v d x-\int_{0}^{a} \phi_{s}(t, x)(\mathbf{u}-\overline{\mathbf{u}})_{x} v_{x} d x=0 \\
(\mathbf{u}-\overline{\mathbf{u}})(0)=0
\end{array}
$$

for each $v \in D_{\psi}$. Setting $v=\mathbf{u}-\overline{\mathbf{u}}$ in (39) we deduce

$$
\frac{1}{2} \frac{d}{d t}\left(\|\mathbf{u}-\overline{\mathbf{u}}\|^{2}\right)=-\int_{0}^{a} \phi_{s}(t, x)\left|(\mathbf{u}-\overline{\mathbf{u}})_{x}\right|^{2} d x \leq 0 .
$$

Integrating (41) over $[0, t]$ and applying (40), we conclude that $\mathbf{u} \equiv \overline{\mathbf{u}}$.

\section{Main results}

\subsection{Existence of solution}

Recall from Theorem (2.3), after $u$ is possibly redefined on a set of measure zero, that (9) implies that $\mathbf{u} \in C\left[0, T ; L^{2}((0, a))\right]$.

Theorem 4.1. Suppose the conditions (6)-(8) hold. Then there exist unique weak solutions to the problems (1)-(3). 
Proof. The proof is split in seven steps.

Step 1. Banach's fixed point theorem will be applied in the space

$$
X:=C\left[0, T ; L^{2}((0, a))\right]
$$

equipped with the norm

$$
\|\mathbf{U}\|_{X}:=\max _{0 \leq t \leq T}\|\mathbf{U}(t)\|_{L^{2}(0, a)}
$$

A fixed point argument to (1)-(3) is

$$
\begin{aligned}
w_{t}-\left(\phi_{u}(t, x) w_{x}\right)_{x} & =f(u), \text { in }(0, T] \times(0, a) \\
w(t, 0) & =0, w(t, a)=0, \text { in }(0, T] \\
w(0, x) & =g(x), x \in(0, a)
\end{aligned}
$$

For a given function $\mathbf{u} \in X$, Theorem 3.4 ensures the existence and uniqueness of the solution to (42)-(44), namely:

$$
\mathbf{w} \in L^{2}\left[0, T ; D_{\psi}\right], \text { with } \mathbf{w}^{\prime} \in L^{2}\left[0, T ; D_{\psi}^{-1}\right] .
$$

Step 2. We define a mapping

$$
M: X \rightarrow X
$$

by setting $M[\mathbf{u}]=\mathbf{w}$ whenever $\mathbf{w}$ is derived from $\mathbf{u}$ via (42)-(44). We will show that $M$ is a contractive mapping for sufficiently small time $T>0$. We choose $\mathbf{u}, \tilde{\mathbf{u}} \in X$ and define $M[\mathbf{u}]=\mathbf{w}, M[\tilde{\mathbf{u}}]=\tilde{\mathbf{w}}$. For two weak solutions $\mathbf{w}, \tilde{\mathbf{w}} \in X$ of (42)-(44), we have

$$
\begin{aligned}
\int_{0}^{a}(\mathbf{w}-\tilde{\mathbf{w}})^{\prime} v+ & \int_{0}^{a} \phi_{\mathbf{u}}(t, x)(\mathbf{w}-\tilde{\mathbf{w}})_{x} v_{x} d x= \\
- & \int_{0}^{a}\left(\phi_{\mathbf{u}}(t, x)-\phi_{\tilde{\mathbf{u}}}(t, x)\right) \tilde{\mathbf{w}}_{x} v_{x} d x+ \\
& \int_{0}^{a}(f(\mathbf{u})-f(\tilde{\mathbf{u}})) v d x \\
(\mathbf{w}-\tilde{\mathbf{w}})(0)= & 0
\end{aligned}
$$

for each $v \in D$. Setting $v=\mathbf{w}-\tilde{\mathbf{w}}$ in (45) we deduce

$$
\begin{aligned}
& \frac{1}{2} \frac{d}{d t}\left(\|\mathbf{w}-\tilde{\mathbf{w}}\|_{L^{2}(0, a)}^{2}\right)+\int_{0}^{a} \phi_{\mathbf{u}}(t, x)(\mathbf{w}-\tilde{\mathbf{w}})_{x}^{2} d x= \\
& -\int_{0}^{a}\left(\phi_{\mathbf{u}}(t, x)-\phi_{\tilde{\mathbf{u}}}(t, x)\right) \tilde{\mathbf{w}}_{x}(\mathbf{w}-\tilde{\mathbf{w}})_{x} d x+\int_{0}^{a}(\mathbf{w}-\tilde{\mathbf{w}})(f(\mathbf{u})-f(\tilde{\mathbf{u}})) d(x 47)
\end{aligned}
$$


Interchanging the roles of $\mathbf{w}$ and $\tilde{\mathbf{w}}$ in (47), we deduce

$$
\begin{aligned}
& \frac{1}{2} \frac{d}{d t}\left(\|\mathbf{w}-\tilde{\mathbf{w}}\|_{L^{2}(0, a)}^{2}\right)+\int_{0}^{a} \phi_{\tilde{\mathbf{u}}}(t, x)(\mathbf{w}-\tilde{\mathbf{w}})_{x}^{2} d x= \\
& -\int_{0}^{a}\left(\phi_{\mathbf{u}}(t, x)-\phi_{\tilde{\mathbf{u}}}(t, x)\right) \mathbf{w}_{x}(\mathbf{w}-\tilde{\mathbf{w}})_{x} d x+\int_{0}^{a}(\mathbf{w}-\tilde{\mathbf{w}})(f(\mathbf{u})-f(\tilde{\mathbf{u}})) d(x 48)
\end{aligned}
$$

Adding (47) and (48) yields:

$$
\begin{aligned}
& \frac{d}{d t}\left(\|\mathbf{w}-\tilde{\mathbf{w}}\|_{L^{2}(0, a)}^{2}\right)+\int_{0}^{a}\left(\phi_{\mathbf{u}}(t, x)+\phi_{\tilde{\mathbf{u}}}(t, x)\right)(\mathbf{w}-\tilde{\mathbf{w}})_{x}^{2} d x= \\
& -\int_{0}^{a}\left(\phi_{\mathbf{u}}(t, x)-\phi_{\tilde{\mathbf{u}}}(t, x)\right)(\mathbf{w}+\tilde{\mathbf{w}})_{x}(\mathbf{w}-\tilde{\mathbf{w}})_{x} d x+ \\
& 2 \int_{0}^{a}(\mathbf{w}-\tilde{\mathbf{w}})(f(\mathbf{u})-f(\tilde{\mathbf{u}})) d x .
\end{aligned}
$$

Applying the condition (6), we deduce

$$
\begin{aligned}
& \frac{d}{d t}\left(\|\mathbf{w}-\tilde{\mathbf{w}}\|_{L^{2}(0, a)}^{2}\right)+2 \lambda_{1} \psi \int_{0}^{a}(\mathbf{w}-\tilde{\mathbf{w}})_{x}^{2} d x \\
& \leq\left(\lambda_{2}-\lambda_{1}\right) \int_{0}^{a} \psi(x)\left|\mathbf{w}_{x}^{2}-\tilde{\mathbf{w}}_{x}^{2}\right|+2 \int_{0}^{a}|\mathbf{w}-\tilde{\mathbf{w}}||f(\mathbf{u})-f(\tilde{\mathbf{u}})| d x \\
& \leq\left(\lambda_{2}-\lambda_{1}\right) \int_{0}^{a} \psi(x)(\mathbf{w}-\tilde{\mathbf{w}})_{x}^{2}+2 \int_{0}^{a}|\mathbf{w}-\tilde{\mathbf{w}}||f(\mathbf{u})-f(\tilde{\mathbf{u}})| d x
\end{aligned}
$$

Since by (6), $3 \lambda_{1}-\lambda_{2}>0,(50)$ can be simplified to get

$$
\begin{aligned}
& \frac{d}{d t}\left(\|\mathbf{w}-\tilde{\mathbf{w}}\|_{L^{2}(0, a)}^{2}\right) \leq 2 \int_{0}^{a}|\mathbf{w}-\tilde{\mathbf{w}}||f(\mathbf{u})-f(\tilde{\mathbf{u}})| d x \\
& \leq 2 L \int_{0}^{a}|\mathbf{w}-\tilde{\mathbf{w}} \| \mathbf{u}-\tilde{\mathbf{u}}|(\text { using }(8)) \\
& \leq L\|\mathbf{w}-\tilde{\mathbf{w}}\|_{L^{2}(0, a)}^{2}+L\|\mathbf{u}-\tilde{\mathbf{u}}\|_{L^{2}(0, a)}^{2} \text { (by Young's inequality). }
\end{aligned}
$$

Gronwall's inequality applied to (51) yields

$$
\begin{aligned}
\|\mathbf{w}(s)-\tilde{\mathbf{w}}(s)\|_{L^{2}(0, a)}^{2} & \leq L e^{L t} \int_{0}^{s}\|\mathbf{u}(t)-\tilde{\mathbf{u}}(t)\|_{L^{2}(0, a)}^{2} d t \\
& \leq C T\|\mathbf{u}-\tilde{\mathbf{u}}\|_{X}^{2}
\end{aligned}
$$

for each $0 \leq s \leq T$. Maximizing the left side of the last inequality yields

$$
\|\mathbf{w}-\tilde{\mathbf{w}}\|_{X}^{2} \leq C T\|\mathbf{u}-\tilde{\mathbf{u}}\|_{X}^{2}
$$


The last inequality implies

$$
\|M[\mathbf{u}]-M[\tilde{\mathbf{u}}]\|_{X} \leq(C T)^{\frac{1}{2}}\|\mathbf{u}-\tilde{\mathbf{u}}\|_{X}
$$

so that $M$ is a strict contraction mapping for $T>0$ sufficiently small such that $(C T)^{\frac{1}{2}}<1$.

STEP 3. Now write $\mathbf{u}^{0}=g$. For $k=0,1,2, \ldots$, inductively define $\mathbf{u}^{k+1} \in$ $Y_{\psi}$, with $\mathbf{u}^{k+1 \prime} \in L^{2}\left[0, T ; D_{\psi}^{-1}\right]$ to be the unique weak solution of the linear boundary value problem:

$$
\begin{aligned}
\frac{\partial}{\partial t}\left(u^{k+1}\right)-\left(\phi_{u^{k}} u_{x}^{k+1}\right)_{x} & =f\left(u^{k}\right), \text { in }(0, T] \times(0, a) \\
u^{k+1}(t, 0) & =0, u^{k+1}(t, a)=0, \text { in }(0, T] \\
u^{k+1}(0, x) & =g(x), x \in(0, a),
\end{aligned}
$$

Notice that Theorem 3.4 justifies our definition of $\mathbf{u}^{k+1}$ as the unique weak solution of (53)-(55). Consequently, by the definition of the mapping $M$, we deduce for $k=0,1,2, \ldots$ :

$$
\mathbf{u}^{k+1}=M\left[\mathbf{u}^{k}\right]
$$

Since $M$ is contractive (for sufficiently small $T>0$ ), there exists $\mathbf{u} \in X$ such that

$$
\lim _{k \rightarrow \infty} \mathbf{u}^{k+1}=\lim _{k \rightarrow \infty} M\left[\mathbf{u}^{k}\right]=M[\mathbf{u}]=\mathbf{u} .
$$

Step 4. Using (23), we deduce

$$
\begin{gathered}
\sup _{0 \leq t \leq T}\left\|\mathbf{u}^{k+1}(t)\right\|_{L^{2}(0, a)}^{2}+\left\|\mathbf{u}^{k+1}\right\|_{L^{2}\left[0, T ; H_{0}^{1}((0, a), \psi(x))\right]}^{2}+\left\|\mathbf{u}^{k+1 \prime}\right\|_{L^{2}\left[0, T ; D_{\psi}^{-1}\right]}^{2} \\
\leq C\left(\|g\|_{L^{2}(0, a)}+\left\|\mathbf{u}^{k}\right\|_{X}+1\right)^{2}
\end{gathered}
$$

from whence using (56) and sending $k$ to infinity on the right side we deduce

$$
\begin{aligned}
\sup _{k}\left\|\mathbf{u}^{k+1}(t)\right\|_{Y_{\psi}} & <\infty, \\
\sup _{k}\left\|\mathbf{u}^{k+1 \prime}\right\|_{L^{2}\left[0, T ; D_{\psi}^{-1}\right]} & <\infty .
\end{aligned}
$$

(58) and (59) imply the existence of a subsequence $\left\{\mathbf{u}^{k_{j}}\right\}_{j=1}^{\infty} \subset\left\{\mathbf{u}^{k}\right\}_{k=1}^{\infty}$ and a function $\mathbf{u} \in Y_{\psi}$, with $\mathbf{u}^{\prime} \in L^{2}\left[0, T ; D_{\psi}^{-1}\right]$, such that

$$
\begin{aligned}
& \mathbf{u}^{k_{j}} \rightarrow \mathbf{u} \text { in } Y_{\psi} \\
& \mathbf{u}^{k_{j} \prime} \rightarrow \mathbf{u}^{\prime} \text { in } L^{2}\left[0, T ; D_{\psi}^{-1}\right]
\end{aligned}
$$

Further, using (17), we deduce

$$
\left\|f\left(\mathbf{u}^{k}\right)\right\|_{L^{2}\left[0, T ; L^{2}(0, a)\right]} \leq C\left(1+\left\|\mathbf{u}^{k}\right\|_{X}\right)
$$


for some constant $C>0$. Using (56), we take the limit on the right side of (62) to conclude

$$
\sup _{k}\left\|f\left(\mathbf{u}^{k}\right)\right\|_{L^{2}\left[0, T ; L^{2}(0, a)\right]}<\infty .
$$

(63) implies the existence of a subsequence $\left\{f\left(\mathbf{u}^{k_{j}}\right)\right\}_{j=1}^{\infty} \in L^{2}\left[0, T ; L^{2}(0, a)\right]$ and function $f(\mathbf{u}) \in L^{2}\left[0, T ; L^{2}(0, a)\right]$ such that

$$
f\left(\mathbf{u}^{k_{j}}\right) \rightarrow f(\mathbf{u}) \text { in } L^{2}\left[0, T ; L^{2}(0, a)\right] .
$$

Step 5. We next verify that $\mathbf{u}$ is a weak solution of (1)-(3). For brevity, we take the subsequence $\left\{\mathbf{u}^{k_{j}}\right\}_{j=1}^{\infty}$ of the last step as the sequence $\left\{\mathbf{u}^{k}\right\}_{k=0}^{\infty}$. Fix $v \in Y_{\psi}$. Since $\mathbf{u}^{k+1}$ is the unique weak solution of (53)-(55), we have

$$
\begin{aligned}
& \int_{0}^{T} \int_{0}^{a} \mathbf{u}^{k+1 \prime} v d x d t+\int_{0}^{T} \int_{0}^{a} \phi_{\mathbf{u}^{k}}(t, x) \mathbf{u}_{x}^{k+1} v_{x} d x d t=\int_{0}^{T} \int_{0}^{a} f\left(\mathbf{u}^{k}\right) v d x d t(64) \\
& \mathbf{u}^{k+1}(0)=g
\end{aligned}
$$

Passage to limit is not immediately apparent in the second term on the left side of (64). Notice that

$$
\begin{aligned}
& \left|\int_{0}^{T} \int_{0}^{a}\left[\phi_{\mathbf{u}^{k}}(t, x) \mathbf{u}_{x}^{k+1}-\phi_{\mathbf{u}}(t, x) \mathbf{u}_{x}\right] v_{x} d x d t\right| \\
& \leq\left|\int_{0}^{T} \int_{0}^{a}\left[\phi_{\mathbf{u}^{k}}(t, x)\left(\mathbf{u}_{x}^{k+1}-\mathbf{u}_{x}\right)+\mathbf{u}_{x}\left(\phi_{\mathbf{u}^{k}}(t, x)(x)-\phi_{\mathbf{u}}(t, x)\right)\right] v_{x} d x d t\right| \\
& \leq \int_{0}^{T}\left(\lambda_{2} \int_{0}^{a} \psi(x)\left|\mathbf{u}_{x}^{k+1}-\mathbf{u}_{x}\right|\left|v_{x}\right| d x+\right. \\
& \leq \int_{0}^{T}\left(\lambda_{2} \int_{0}^{a} \psi(x)\left|\mathbf{u}_{x}^{k+1}-\mathbf{u}_{x}\right|\left|v_{x}\right| d x+\right. \\
& \left.L_{1}^{a} \int_{0}^{a}\left|\mathbf{u}^{k}-\mathbf{u}\right| d x \int_{0}^{a} \psi(x)\left|\mathbf{u}_{x}\right|\left|v_{x}\right| d x\right) d t \\
& \leq\left(\lambda_{2}\left\|\mathbf{u}^{k+1}-\mathbf{u}\right\|_{Y_{\psi}}+L_{1}\|\mathbf{u}\|_{Y_{\psi}}\left\|\mathbf{u}^{k}-\mathbf{u}\right\|_{Y_{\psi}}\right)\|v\|_{Y_{\psi}} \rightarrow 0 \text { as } k \rightarrow \infty \text { (Using (6) and (7)) }
\end{aligned}
$$

Hence, using (60), (61), the deduction on $\left\{f\left(\mathbf{u}^{k_{j}}\right)\right\}_{j=1}^{\infty}$ in the last step and (66), we send $k \rightarrow \infty$ in (64)-(65) to get

$$
\begin{aligned}
\int_{0}^{T} \int_{0}^{a} \mathbf{u}^{\prime} v d x d t+\int_{0}^{T} \int_{0}^{a} \phi_{\mathbf{u}}(t, x)(x) \mathbf{u}_{x} v_{x} d x d t & =\int_{0}^{T} \int_{0}^{a} f(\mathbf{u}) v d x d t \\
\mathbf{u}(0) & =g
\end{aligned}
$$


from whence we deduce (10)-(11) as desired.

Step 6 . For any given $T>0$, we select $T_{1}>0$ sufficiently small that $\left(C T_{1}\right)^{\frac{1}{2}}<$ 1. Banach's fixed point theorem can be applied to find a weak solution $\mathbf{u}$ of the problem (1)-(3) on the time interval $\left[0, T_{1}\right]$. Since $\mathbf{u}(t) \in Y_{\psi}$ for a.e. $0 \leq t \leq T$, we can upon redefining $T_{1}$ if necessary assume that $\mathbf{u}\left(T_{1}\right) \in Y_{\psi}$. The argument above can be used to extend our solution to the time interval $\left[T_{1}, 2 T_{1}\right]$. We continue after finitely many steps to construct a weak solution of (1)-(3) existing on the whole interval $[0, T]$.

Step 7. Lastly, we prove uniqueness. Suppose there exists two weak solutions $\mathbf{u}$ and $\tilde{\mathbf{u}}$ of $(1)-(3)$. Then we have $\mathbf{w}=\mathbf{u}, \tilde{\mathbf{w}}=\tilde{\mathbf{u}}$ in (51); so that

$$
\frac{d}{d t}\left(\|\mathbf{u}(t)-\tilde{\mathbf{u}}(t)\|_{L^{2}(0, a)}\right) \leq 2 L\|\mathbf{u}(t)-\tilde{\mathbf{u}}(t)\|_{L^{2}(0, a)}
$$

for $t \in[0, T]$. Gronwall's inequality applied to (69) implies that $\mathbf{u} \equiv \tilde{\mathbf{u}}$.

\subsection{Nonexistence or blow-up of solution}

Theorem 4.2. Let the conditions (4) and (6) hold. Suppose that $T>0$ and $g \geq 0$ are large enough in an appropriate sense, then, either there does not exist a smooth solution $u$ of (1)-(3) or the solution blows up in a finite time.

Proof. Let $w_{1}$ be an eigenfunction corresponding to the principal eigenvalue $\mu_{1}>0$ of $-\frac{\partial}{\partial x}\left(\psi(x) \frac{\partial}{\partial x}\right)$ in $H_{0}^{1}((0, a), \psi(x))$. Then $w_{1}$ is smooth [10]. We have

$$
\begin{aligned}
-\left(\psi(x) w_{1 x}\right)_{x} & =\mu_{1} w_{1} \text { in }(0, a) \\
w_{1}(0)=0, w_{1}(a) & =0
\end{aligned}
$$

We may further assume, without loss of generality, that

$$
w_{1}>0 \text { in }(0, a), \int_{0}^{a} w_{1} d x=1
$$

Suppose $u$ is a smooth solution of (1)-(3), with $g \geq 0, g \not \equiv 0$. Then $u>0$ within $(0, T] \times(0, a)$ by the strong maximum principle. We define

$$
\eta(t):=\int_{0}^{a} u(t, x) w_{1}(x) d x \quad(0 \leq t \leq T) .
$$


Hence

$$
\begin{aligned}
\dot{\eta}(t)= & \int_{0}^{a} u_{t} w_{1} d x=\int_{0}^{a}\left[\left(\phi_{u}(t, x) u_{x}\right)_{x}+f(u)\right] w_{1} d x, \quad \text { (using (1.1)) } \\
= & -\int_{0}^{a} \phi_{u}(t, x) u_{x} w_{1 x} d x+\int_{0}^{a} f(u) w_{1} d x, \quad \text { integrating by parts) } \\
= & -\left[\int_{0}^{a} \phi_{u}(t, x)\left[u_{x} w_{1 x}\right]^{+} d x-\int_{0}^{a} \phi_{u}(t, x)\left[u_{x} w_{1 x}\right]^{-} d x\right]+\int_{0}^{a} f(u) w_{1} d x \\
\geq & -\left[\int_{0}^{a} \beta \psi(x)\left[u_{x} w_{1 x}\right]^{+} d x-\int_{0}^{a} \beta \psi(x)\left[u_{x} w_{1 x}\right]^{-} d x\right]+\int_{0}^{a} f(u) w_{1} d x \\
& (\operatorname{using}(5)) \\
= & -\beta \int_{0}^{a} \psi(x) u_{x} w_{1 x} d x+\int_{0}^{a} f(u) w_{1} d x \\
= & \beta \int_{0}^{a} u\left(\psi(x) w_{1 x}\right)_{x} d x+\int_{0}^{a} f(u) w_{1} d x, \quad(\text { integrating by parts }) \\
\geq & -\beta \mu_{1} \int_{0}^{a} u w_{i} d x+\int_{0}^{a} \gamma u^{p} w_{1} d x=-\beta \mu_{1} \eta(t)+\gamma \int_{0}^{a} u^{p} w_{1} d x,
\end{aligned}
$$

(using (4) and (70))

where $v^{+}:=\max (v, 0)$ and $v^{-}:=\min (v, 0)$. For $1<p, q<\infty$ with $\frac{1}{p}+\frac{1}{q}=1$, we have by Hölder's inequality:

$$
\begin{aligned}
\eta(t)=\int_{0}^{a} u w_{1} d x & =\int_{0}^{a} u w_{1}^{\frac{1}{p}} w_{1}^{\frac{1}{q}} \leq\left(\int_{0}^{a} u^{p} w_{1} d x\right)^{\frac{1}{p}}\left(\int_{0}^{a} w_{1} d x\right)^{\frac{1}{q}} \\
& =\left(\int_{0}^{a} u^{p} w_{1} d x\right)^{\frac{1}{p}} \quad(\text { by }(72)) .
\end{aligned}
$$

(74) implies that

$$
\eta(t)^{p} \leq \int_{0}^{a} u^{p} w_{1} d x .
$$

Using the last inequality in (73), we have

$$
\dot{\eta}(t) \geq-\beta \mu_{1} \eta(t)+\gamma \eta(t)^{p} \quad(0 \leq t \leq T) .
$$

Using $\zeta(t):=\eta(t)^{1-p},(75)$ transforms to

$$
\dot{\zeta}(t)-(p-1) \beta \mu_{1} \zeta(t) \leq-\gamma(p-1)
$$

Multiplying (76) by $e^{-(p-1) \beta \mu_{1} t}$, we deduce

$$
\left(e^{-(p-i) \beta \mu_{1} t} \zeta\right) \leq-e^{-(p-1) \beta \mu_{1} t} \gamma(p-1),
$$


which on integrating one deduces

$$
\begin{aligned}
\zeta & \leq e^{(p-1) \beta \mu_{1} t}\left[\zeta(0)-\frac{\gamma}{\beta \mu_{1}}\left(1-e^{-(p-1) \beta \mu_{1} t}\right)\right] \\
& \leq e^{(p-1) \beta \mu_{1} t}\left[\frac{1}{\eta(0)^{p-1}}-\frac{\gamma}{\beta \mu_{1}}\left(1-e^{-(p-1) \beta \mu_{1} t}\right)\right] \\
& =e^{(p-1) \beta \mu_{1} t}\left[\frac{\beta \mu_{1}-\gamma \eta(0)^{p-1}\left(1-e^{-(p-1) \beta \mu_{1} t}\right)}{\beta \mu_{1} \eta(0)^{p-1}}\right]
\end{aligned}
$$

Hence

$$
\eta(t)^{p-1}=\frac{1}{\zeta(t)} \geq e^{-(p-1) \beta \mu_{1} t}\left[\frac{\beta \mu_{1} \eta(0)^{p-1}}{\beta \mu_{1}-\gamma \eta(0)^{p-1}\left(1-e^{-(p-1) \beta \mu_{1} t}\right)}\right]
$$

Consequently,

$$
\eta(t) \geq \frac{e^{-\beta \mu_{1} t}\left(\beta \mu_{1}\right)^{\frac{1}{p-1}} \eta(0)}{\left[\beta \mu_{1}-\gamma \eta(0)^{p-1}\left(1-e^{-(p-1) \beta \mu_{1} t}\right)\right]},
$$

provided the denominator is not zero. Suppose now that

$$
\eta(0)>\left(\frac{\beta \mu_{1}}{\gamma}\right)^{\frac{1}{p-1}}
$$

then

$$
\eta(t) \rightarrow+\infty \quad \text { as } \quad t \rightarrow t^{*}
$$

where

$$
t^{*}:=\frac{1}{(p-1) \beta \mu_{1}} \log \left(\frac{\gamma \eta(0)^{p-1}}{\gamma \eta(0)^{p-1}-\beta \mu_{1}}\right) .
$$

Thus, if

$$
\eta(0)=\int_{0}^{a} g w_{1} d x>\left(\frac{\beta \mu_{1}}{\gamma}\right)^{\frac{1}{p-1}}
$$

then there does not exist a smooth solution $u$ of (1)-(3). Either the solution is not smooth enough to justify the calculation above; or

$$
\lim _{t_{*} \rightarrow t^{*}} \int_{0}^{a} u(x, t) w_{1} d x=\infty
$$

for some $0<t_{*} \leq t^{*}$; in which case the solution blows-up. 


\section{$5 \quad$ Illustrative examples}

Example 5.1. Consider the problem

$$
\begin{aligned}
u_{t}-\left(x^{\alpha}\left[3 a+\int_{0}^{x}(3+\sin u) d s\right] u_{x}\right)_{x} & =\sqrt{1+u^{2}}, \text { in }(0, T] \times(0, a), a \geq \frac{1}{3}(78) \\
u(t, 0) & =0, u(t, a)=0, \text { in }(0, T] \\
u(0, x) & =x^{2}, x \in(0, a)
\end{aligned}
$$

where $\alpha$ is any arbitrary positive number. Notice that we have $\phi_{u}(t, x):=$ $x^{\alpha}\left[3 a+\int_{0}^{x}(3+\sin u) d s\right] \geq 0$ satisfies (6) and (7) with $\psi(x):=x^{\alpha}, \lambda_{1}:=3 a \geq$ $1, \lambda_{2}:=7 a, 3 \lambda_{1}-\lambda_{2}=2 a>0, L_{1}:=1$; and the nonsmooth nonlinearity $f(u):=\sqrt{1+u^{2}}$ is Lipschitz continuous (with $L=1$ ) and $g(x):=x^{2} \in$ $L^{2}(0, a)$. Theorem 4.1 ensures the existence and uniqueness of the solution to (78)-(80).

Example 5.2. We next consider

$$
\begin{aligned}
u_{t}- & \left((1-|\cos (20 \pi x / a)|)\left[a+\int_{0}^{x} \frac{\sqrt{u^{2}+1}}{1+\sqrt{u^{2}+1}} d s\right] u_{x}\right)_{x}= \\
& 2|u|, \text { in }(0, T] \times(0, a) \\
& u(t, 0)=0, u(t, a)=0, \text { in }(0, T] \\
& u(0, x)=\sin x, x \in(0, a),
\end{aligned}
$$

where $a \geq 1$. We have that $\phi_{u}(t, x):=(1-|\cos (20 \pi x / a)|)\left[a+\int_{0}^{x} \frac{\sqrt{u^{2}+1}}{1+\sqrt{u^{2}+1}} d s\right] \geq$ 0 satisfies (6) and (7) with $\psi(x):=(1-|\cos (20 \pi x / a)|), \lambda_{1}:=a \geq 1, \lambda_{2}:=$ $2 a, 3 \lambda_{1}-\lambda_{2}=a>0, L_{1}:=1 ; f(u):=2|u|$ is Lipschitz continuous (with $L=2)$ and $g(x):=\sin x \in L^{2}(0, a)$. The existence and uniqueness of solution to (81)-(83) is guaranteed by Theorem 4.1. Notice that (81) is not only degenerate at the end points, but also at some points within $(0, a)$.

Example 5.3. We next give an example where there does not exist a smooth solution or where the solution blows up. Consider the problem

$$
\begin{aligned}
\left.u_{t}-\int_{0}^{x}(3+\sin u) d s u_{x}\right)_{x} & =\sqrt{1+u^{4}}, \text { in }(0, T] \times(0,1) \\
u(t, 0) & =0, u(t, 1)=0, \text { in }(0, T] \\
u(0, x) & =6 \pi^{3}, x \in(0,1)
\end{aligned}
$$

Notice that we have that $\phi_{u}(t, x):=\int_{0}^{x}(3+\sin u) d s \geq 0$ satisfies (5) with $\psi(x):=1, \beta:=4$; and the nonsmooth nonlinearity $f(u):=\sqrt{1+u^{4}}$ satisfies (4) with $\gamma:=1, p:=2>1 . g(x):=6 \pi^{3} \in L^{2}(0,1)$. Now $w_{1}:=\frac{\pi}{2} \sin \pi x$ is 
an eigenfunction corresponding to the principal eigenvalue $\mu_{1}:=\pi^{2}$ of $-u^{\prime \prime}$ in $H_{0}^{1}(0,1)$. Notice that $w_{1}$ is smooth and that

$$
w_{1}>0 \text { in }(0,1), \quad \int_{0}^{1} w_{1} d x=1
$$

Suppose $u$ is a smooth solution of (84)-(86). Since $g>0$, we have $u>0$ within $(0, T] \times(0,1)$ by the strong maximum principle. Define

$$
\eta(t):=\int_{0}^{1} u(t, x) w_{1} d x=\int_{0}^{1} u(t, x)\left(\frac{\pi}{2} \sin \pi x\right) d x \quad(0 \leq t \leq T) .
$$

Using (77), we have

$$
\eta(0)=\int_{0}^{1} g w_{1} d x=\int_{0}^{1} 6 \pi^{3}\left(\frac{\pi}{2} \sin \pi x\right) d x=6 \pi^{2}>\frac{\beta \mu_{1}}{\gamma}=4 \pi^{2} .
$$

Consequently, either there cannot exist a smooth solution of (84)-(86); or else

$$
\lim _{t_{*} \rightarrow t^{*}} \int_{0}^{1} u(x, t)\left(\frac{\pi}{2} \sin \pi x\right) d x=\infty
$$

for some $0<t_{*} \leq t^{*}$, where

$$
t^{*}:=\frac{1}{4 \pi^{2}} \log 3
$$

in which case the solution blows-up in a finite time.

\section{References}

[1] K. B. Athreya and S. N. Lahiri, Measure theory and probability theory, Springer, 2006.

[2] A. Bose and G. A. Kriegsmann, Stability of localized structures in nonlocal reaction-diffusion equations, Methods Appl. Anal. 5 (1998), No. 4, 351-366.

[3] C. Budd, J. Dold and A. Stuart, Blow-up in a partial differential equation with constrained first integral, SIAM J. Appl. Math., 53 (1993), 718-742.

[4] C. Budd, V. A. Galaktionov and J. Chen, Focusing blow-up for qusilinear parabolic equations, Proc. Roy. Soc. Edinb., 128A (1998), 965-992. 
[5] A. Calsina, C. Perello and J. Saldana, Non-local reaction-diffusion equations modelling predator-prey coevolution, Publ. Mat. 389 (1994), 315325 .

[6] T. Cazenave and P. L. Lions, Solutions globales d'équations de la chaleur semilinéares, Comm. Partial Differential Equations 9 (1984), 955-978.

[7] C. Y. Chan, H. T. Liu, Global existence of solutions for degenerate semilinear parabolic equations, Nonlinear Anal., 34 (1998), 617-628.

[8] Y. Chen, Q. Liu, and C. Xie, Blow-up for degenerate parabolic equations with nonlocal source, Proc. Amer. Math. Soc., 132 (2003), No. 1, 135-145.

[9] Y. Chen and L. Lihua, Boundedness of global positive solutions of a localized degenerate parabolic equation, J. Adv. Res. Differ. Eqn. 4 (2011), No. 1, 63-80.

[10] L. C. Evans, Partial Differential Equations, American Mathematical Society, Providence, Rhode Island, 1998.

[11] M. S. Floater, Blow up at the boundary for degenerate semilinear parabolic equations, Arch. Rat. Mech. Anal., 114 (1991), 57-77.

[12] A. Friedman and B. Mcleod, Blow-up of positive solutions of semilinear heat equations, Indiana Univ. Math. J. 34 (1985), 425-447.

[13] Y. Giga and R.V. Kohn, Asymptotic self-similar blow-up of semilinear heat equations, Comm. Pure Appl. Math. 38 (1985), 297-319.

[14] C. Mu, D. Liu and S. Zhou, Properties of positive solutions for a nonlocal reaction-diffusion equation with nonlocal nonlinear boundary condition, J. Korean Math. Soc. 47 (2010), No. 6, 1317-1328.

[15] W. M. Ni, P. E. Sacks and J. Tavantzis, On the asymptotic behavior of solutions of certain quasilinear parabolic equations, J. Differential Equations 54 (1984), 97-120.

[16] C. Peng, Z. Yang and B. Xie, Global existence and blow-up for the degenerate and singular nonlinear parabolic system with a nonlocal source, Nonlinear Anal. 72 (2010), No. 5, 2474-2487.

[17] J. Rubinstein and P. Sternberg, Nonlocal reaction-diffusion equations and nucleation, IMA J. Appl. Math. 48 (1992), No. 3, 249-264.

[18] S. A. Sanni, Global unique weak solutions of nonlocal degenerate reactiondiffusion equations in weighted Sobolev's spaces. To appear. 
[19] P. Souplet, Blow-up in Nonlocal Reaction-Diffusion Equations, SIAM J. Math. Anal. 29 (1998), No. 6, 1301-1334.

[20] P. Souplet, Uniform blow-up profile and boundary behavior for diffusion equations with nonlocal nonlinear source, J. Differential Equations, 153 (1999), 374-406.

[21] Y. Wang, C. Mu, and Z. Xiang, Properties of Positive Solution for Nonlocal Reaction-Diffusion Equation with Nonlocal Boundary, Bound. Value Probl. 2007 (2007), Article ID 64579, 1-12.

[22] P. Weng and X-Q. Zhao, Spatial dynamics of a nonlocal and delayed population model in a periodic habitat, Discrete Contin. Dyn. Syst. 29 (2011), No. 1, 343-366.

\section{Received: August, 2012}

